\title{
GENERATION OF ULTRA-REALISTIC SYNTHETIC ECHOCARDIOGRAPHIC SEQUENCES TO FACILITATE STANDARDIZATION OF DEFORMATION IMAGING
}

\author{
M. Alessandrini ${ }^{\star}$, B. Heyde ${ }^{\star}$, S. Giffard-Roisin ${ }^{\wedge}$, H. Delingette ${ }^{\wedge}$, M. Sermesant ${ }^{\wedge}$, \\ P. Allain*, O. Bernard ${ }^{\diamond}$, M. De Craene* and J. D’hooge \\ ${ }^{\star}$ Cardiovascular Imaging and Dynamics, KU Leuven, ${ }^{\wedge}$ Asclepios Project, INRIA, \\ ${ }^{\diamond}$ CREATIS, CNRS UMR5220, Inserm U1044, INSA-Lyon, ${ }^{*}$ Medisys, Philips Research Paris;
}

\begin{abstract}
Despite the overwhelming availability of techniques for computation of cardiac deformation and strain with $2 \mathrm{D}$ echocardiography, their widespread dissemination in clinical practice is still held back by the reported low reproducibility between different solutions. This can in part be attributed to the lack of a solid and open quality assurance framework to assess and compare their performance. Building upon our previous work, we present here a new pipeline for the benchmarking of such algorithms making use of realistic 2D synthetic sequences. While the synthetic motion is fully controlled by a computational heart model, the visual appearance is extremely similar to a real ultrasound recording. The pipeline is used to generate an initial library of four sequences.
\end{abstract}

Index Terms - echocardiography, simulation, cardiac deformation, standardization.

\section{INTRODUCTION}

Echocardiography is the modality of choice in clinical diagnostics for the assessment of heart function. Besides global indexes as ejection fraction, the analysis of regional cardiac deformation/strain, in particular in the longitudinal direction (i.e. apex to base), is raising increasing clinical interest due to the higher sensitivity and specificity for the early diagnosis of conditions such as ischemia and dyssynchrony [1]. Hereto, a variety of techniques (speckle tracking echocardiography, STE) is available for the quantification of cardiac deformation and strain by ultrasound (US). Although added diagnostic value has been proven, major clinical concern is raised by the poor reproducibilty between vendors [1]. To address this issue, a task force of the European Association of Cardiovascular Imaging (EACVI), the American Society of Echocardiography (ASE) and industry was recently established [2]. Although 3D US faces the same pitfalls [1], its employment in clinical practice is still fairly limited. Therefore, the compelling clinical concern is to date directed to 2D US [2].

The main factor for low reproducibility is the lack of a solid quality assurance protocol for STE. In this perspective,

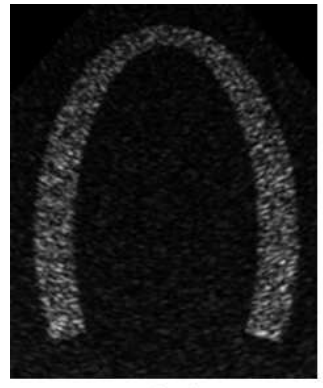

(a)

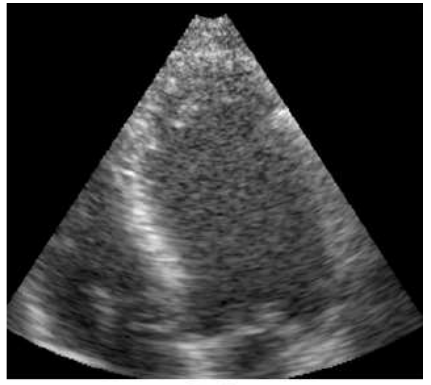

(b)
Fig. 1. Example image used in the EACVI/ASE standardization effort [6] (a) and example of an image generated with the methodology proposed in this paper (b).

the employment of synthetic datasets has been proven useful for a preliminary evaluation in silico [3]: it does not require complex setups (unlike animal models or cardiac phantoms), it provides the ground truth deformation pixelwise and allows direct evaluation (i.e. no co-registration with a different modality such as sonomicrometry or MRI is necessary) [5]. Unfortunately, the level of realism of existing simulation techniques is highly unsatisfactory: surrounding structures such as papillary muscles and heart valves are typically neglected as well as typical artifacts such as reverberations, clutter noise, signal dropout and local intensity variations due to changing cardiac fiber orientation (cf. Fig. 1).

In this context, our latest efforts targeted the development of more reliable simulation techniques [4,5]. In particular, most recently we have developed a pipeline to simulate realistic 3D echocardiographic sequences [5]. The pipeline combined most recent advances in the field of electromechanical (E/M) modeling [7] and ultrasound simulation [6] within a novel scheme where a real 3D recording was used as a template for speckle texture. As such, synthetic datasets were generated where cardiac motion was fully controlled by the E/M model while visual appearance was extremely similar to a real acquisition.

In this paper, we present a modification of that pipeline to 


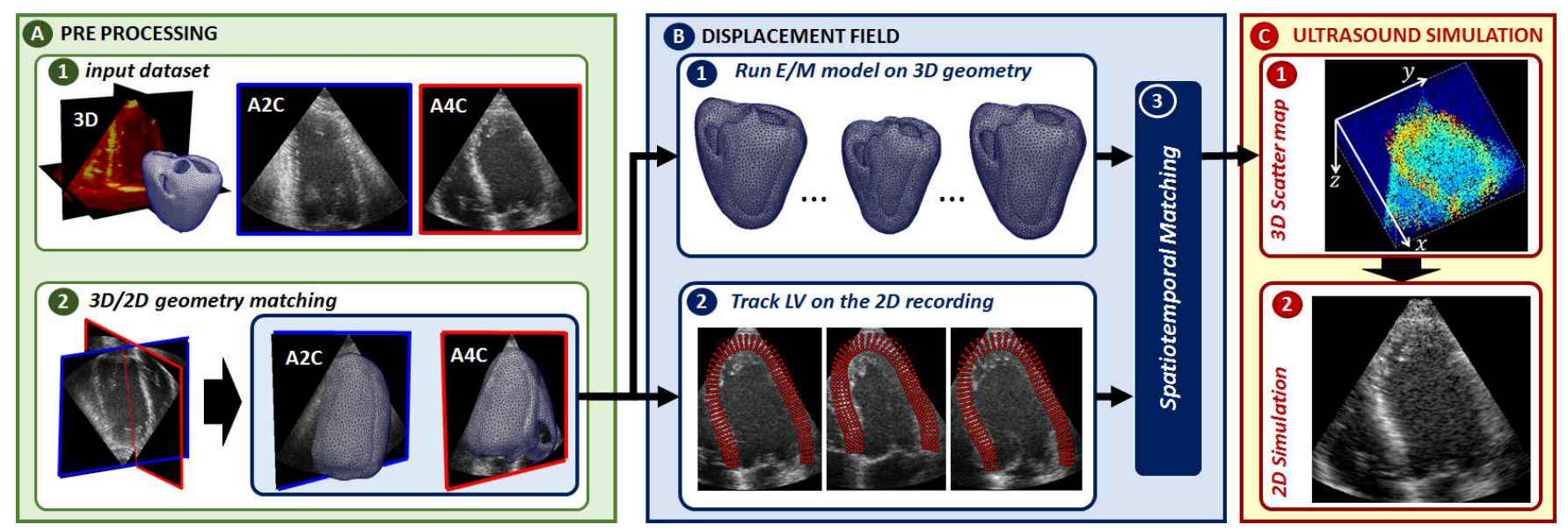

Fig. 2. Proposed simulation pipeline.

generate realistic 2D simulations. We note that simple slicing simulated 3D volumes obtained with [5] would not produce representative 2D simulations since $i$ ) the field of view in 2D is typically wider, $i i$ ) the image quality (i.e. spatial resolution) is typically higher. To account for these differences, we propose a modification of [5] that employs a $2 \mathrm{D}$ recording as model for speckle texture. This modification introduces additional challenges w.r.t. [5] such as to register the 2D acquisition with the 3D E/M geometry and to define the 2D ground truth displacement from the 3D motion of the E/M model. The new pipeline is used to generate simulated apical four chamber (A4C) and apical two chamber (A2C) acquisitions from a healthy and ischemic heart.

\section{MATERIALS AND METHODS}

A schematic of the pipeline is summarized in Fig. 2, which will be used to structure the remainder of this section. Briefly, a real 2D recording is used as template for speckle texture (A.1). The first step is to obtain a 3D heart geometry aligned with the first frame of the 2D template (A.2). The E/M model is then applied to the 3D geometry to simulate one cardiac cycle (B.1). The E/M simulation determines the position of the myocardium in the simulation space. On the other side (B.2), the LV position is tracked over time on the 2D recording. A displacement field is computed (B.3) to align the two datasets (i.e. the 3D E/M simulation and the $2 \mathrm{D}$ recording) in time (by matching cardiac events) and space (by matching the $3 \mathrm{D}$ geometry with the $2 \mathrm{D}$ tracking). The displacement field is then used to move a 3D set of point scatterers (C.1). Scattering amplitude is sampled from the matched position on the $2 \mathrm{D}$ recording. Finally, the scatter map is fed to the ultrasound simulator to generates the 2D synthetic images (C.2).

\subsection{A - Preprocessing}

A.1: On the same healthy volunteer, one 3D and two 2D (one $\mathrm{A} 4 \mathrm{C}$ and one $\mathrm{A} 2 \mathrm{C}$, frame rate $\sim 50 \mathrm{~Hz}$ ) cine scans (one full cycle from end of diastole, ED) were acquired with a GE Vivid 7 system equipped with a MS4 probe. From the first frame of the 3D scan right and left ventricles were segmented manually. From the segmentation result a tetrahedral mesh was obtained with the software TetGen (www.tetgen.org/). A.2: The first frames of the A4C and $\mathrm{A} 2 \mathrm{C}$ scans were then registered with the first frame of the $3 \mathrm{D}$ volume. This was done manually by an expert cardiologist in two steps. By navigating through the 3D dataset, the slice giving the best visual match with the $2 \mathrm{D}$ acquisition was first selected. 3D navigation was performed with an in-house developed visualization interface (Speqle3D [8]). Then, to account for different probe positions, the selected 3D slice and the $2 \mathrm{D}$ view were registered rigidly by using anatomical landmarks (apex and mitral annulus) identified on the two images. After this procedure, the 2D sequences and the 3D cardiac anatomy were aligned in the same space.

\subsection{B - Motion Field}

From the $2 \mathrm{D}$ recording aligned with the $3 \mathrm{D}$ geometry, two further outputs were generated. B.1: At first, the E/M model in [7] and implemented in the SOFA framework (http://www.sofa-framework.org/) was applied to the 3D anatomy to generate two simulations of healthy and ischemic cases. Simulated time resolution was $50 \mathrm{~Hz}$. The ischemic case was produced by altering the contractility of segments perfused by the left circumflex artery (LCX) [9]. B.2: The segmented 3D geometry was used to define on the first frame of the 2D recording a set of node points, which were then tracked over time on the $2 \mathrm{D}$ sequence with the elastic registration technique in [10]. The set of node points served to identify at each time the LV in the $2 \mathrm{D}$ template 
recording. B.3: The two outputs were used to match in space and time the 3D simulation and the $2 \mathrm{D}$ template recording. Temporal synchronization was performed by linearly stretching/shrinking the time axes so to align relevant cardiac phases (systole, i.e. from $\mathrm{ED} 1$ to $\mathrm{ES}$, and diastole, i.e. from $\mathrm{ES}$ to $\mathrm{ED} 2$, were chosen in particular) on the two sequences. As such, each simulation time $t_{\text {sim }}$ was assigned a matched time instant $t_{i m}$ in the 2D recording.

One displacement field $\mathcal{I}_{0 \rightarrow t_{i m}}\left(\mathbf{x}_{2 D}\right)$ was then computed to match coordinates between frames 0 and $t_{i m}$ on the 2D template recording. Similarly, the transform $\mathcal{S}_{t_{s i m} \rightarrow 0}\left(\mathbf{x}_{3 D}\right)$ was computed to match the simulation space at times $t_{\text {sim }}$ and 0 . Subscript $3 D$ and $2 D$ were used to discriminate the $3 D$ simulation space from the $2 D$ image space. In our implementation, both mappings were computed from a Thin Plate Spline (TPS) transform parametrized by the 2D tracking seed points in the case of $\mathcal{I}$ and the $3 \mathrm{D} \mathrm{E} / \mathrm{M}$ mesh nodes in the case of $\mathcal{S}$.

\subsection{C - Ultrasound Simulation}

C.1: The first step was to generate a scatter map by randomly distributing $N$ point scatterers on the first frame of the $2 \mathrm{D}$ template and scattering amplitude was obtained by sampling the image intensity. A $3^{\text {rd }}$ dimension $z$ with random distribution was added to the scatterers coordinates so to cover a thickness of $\sim 3 \mathrm{~cm}$ around the image plane. The reason for this will become clearer later on. As the 3D E/M mesh matches the first image frame, it can be used to differentiate foreground (within the myocardium) from background scatterers. Barycentric E/M mesh coordinates of foreground scatterers were computed and stored.

For generating the scatter map at time $t_{\text {sim }}>0$, foreground scatterers were moved according to the E/M meshes by converting the barycentric coordinates computed on the first frame to Cartesian. Their amplitude was kept constant over time to have speckle coherency in the simulated myocardium. Background scatterers were redrawn randomly at each time excluding the myocardial volume. To have a realistic appearance of the surrounding background, the amplitude of background scatterers was obtained by sampling the intensity of the 2D template recording at the appropriate time and position, as given by the computed spatiotemporal matching. Namely, for each background scatterer $\mathbf{x}_{3 D}^{s i m}$ at time $t_{\text {sim }}$ its matched position at time 0 was computed as $\mathbf{x}_{3 D}^{0}=\mathcal{S}_{t_{s i m} \rightarrow 0}\left(\mathbf{x}_{3 D}^{s i m}\right)$. Its projection $\mathbf{x}_{2 D}^{0}$ on the image plane was computed by dropping the $z$ coordinate. The mirrored position of $\mathbf{x}_{3 D}^{s i m}$ on the associated $2 \mathrm{D}$ template frame at $t_{i m}$ was then given by $\mathbf{x}_{2 D}^{i m}=\mathcal{I}_{0 \rightarrow t_{i m}}\left(\mathbf{x}_{2 D}^{0}\right)$. Finally, the scattering amplitude of $\mathbf{x}_{3 D}^{s i m}$ was computed as $a=I_{t_{i m}}\left(\mathbf{x}_{2 D}^{i m}\right)$, being $I_{t_{i m}}$ the intensity of the template frame at time $t_{i m}$.

C.2: The 3D scatter map was set as the input of a fast ultrasound simulator [6] that computes radio-frequency signals and further performs envelope detection and scan con-
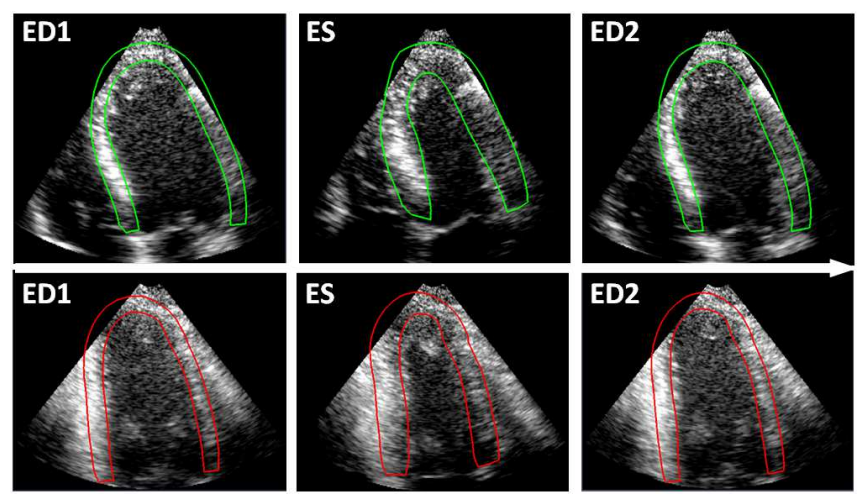

Fig. 3. Example frames from the A4C (top row) and A2C (bottom row) simulations. The bold contour denotes the benchmark LV position. Videos showing the full simulated sequences can be downloaded at http: / / bit. ly/1qmeFbt.

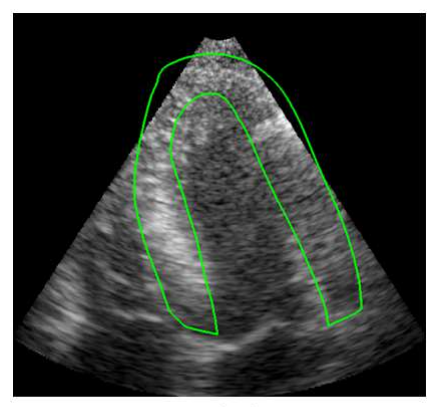

(a)

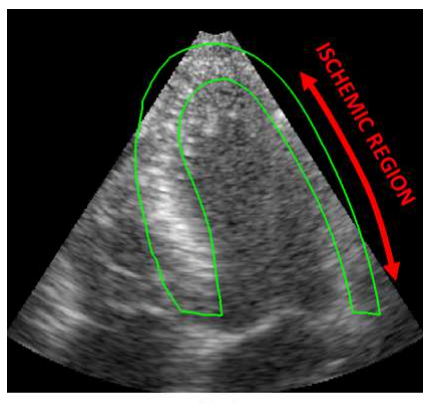

(b)
Fig. 4. Comparison between end-systolic frames from healthy (a) and LCX (b) simulation (A4C view). Note the reduced mobility of the diseased region.

version. A 1D phased array was defined to provide $2 \mathrm{D}$ images. Assigning the scatter map a dummy thickness, instead of defining a single layer of scatterers, was necessary to account for the possible out-of-plane motion imposed by the 3D E/M model.

\subsection{Benchmark displacement/strain}

The reference displacement/strain was computed from a set of seed points sampling the LV myocardium regularly in the longitudinal and radial directions (cf. Fig. 5). The points were displaced according to the ground truth E/M meshes and re-projected on the image plane at each time step. As recommended in [2], radial and longitudinal strain $\epsilon_{R}$ and $\epsilon_{L}$ were then measured by the relative change in distance between neighboring points. Namely, $\epsilon_{n}(k)=d_{n}^{k} / d_{n}^{0}-1$ with $d_{n}^{k}$ the distance between two consecutive points along direction $n=\{R, L\}$ at time $k$. As in [10], drift correction was applied to bring strain curves to zero at the end of the cycle. Point estimates can be averaged globally to obtain ground truth global longitudinal strain (GLS) and global radial strain 


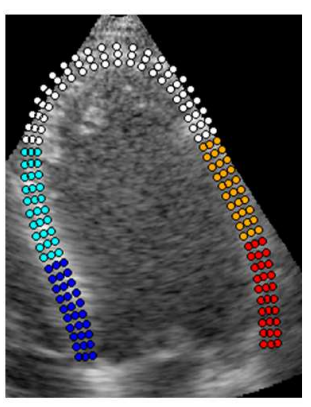

(a) segments

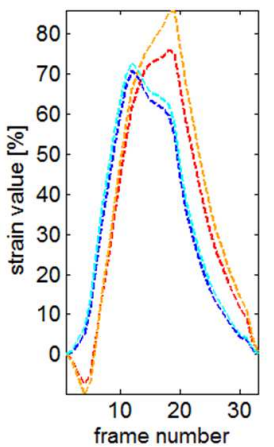

(b) healthy

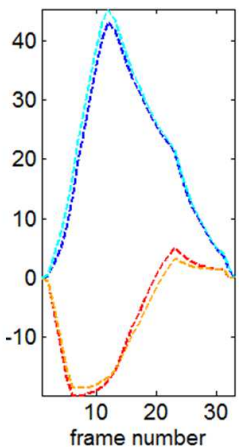

(c) ischemic
Fig. 5. Segments definition (a): basal and mid inferoseptal (blue and cyan), apex (white), mid and basal anterolateral (orange and red). Segmental curves of radial strain for healthy (b) and ischemic (c) simulations.

(GRS), per layer to obtain strain at endo-/epicardium and mid wall or per cardiac segment [5] to obtain segmental strain curves. These quantities can be used to benchmark STE algorithms.

\section{RESULTS}

Example frames from the $\mathrm{A} 4 \mathrm{C}$ and $\mathrm{A} 2 \mathrm{C}$ healthy simulations are reported in Fig. 3. The realism of this simulated data has to be compared with state of the art strategies, as plotted in Fig. 11 The inclusion of a real clinical recording in the pipeline adds realistic challenges for motion tracking: presence of pericardium, valves, etc. As such, the pipeline provides representative datasets for the reliable evaluation of $2 \mathrm{D}$ STE algorithms in silico. Moreover, in combination with [5], the same E/M simulation can be used to simulate realistic 2D and corresponding 3D acquisitions. Therefore, a rigorous quantitative evaluation of the pitfalls of 2D US as compared to $3 \mathrm{D}$ becomes possible.

Speckle texture inside the myocardium is realistic and can be adapted to several probe designs [6]. Motion within the myocardium is unbiased to any image registration algorithm. Although two cases (healthy and ischemic) have been illustrated here, several intermediate cases can be generated to study the sensitivity of any image-based strain quantification technique under a controlled and reproducible environment.

Ischemic segments have reduced mobility as compared to healthy ones Fig. 4. The ground truth radial strain curves available from the healthy and LCX E/M simulations for the A4C view are reported in Fig. 5. Note that regional strain curves correctly identify ischmic segments (red and orange).

\section{CONCLUSION}

Building on our previous work, we proposed a framework to simulate highly realistic $2 \mathrm{D}$ echocardiographic sequences which represents an important shift with respect to the stateof-the-art. The initial database will be expanded to span a wider range of image qualities, probe orientations and pathological conditions and will be made available to the research community. Our effort is to provide a new generation of tools for quality assurance of software packages for the analysis of cardiac function with 2D and 3D US [5]. We aim to integrate our efforts with the ongoing EACVI/ASE standardization task force.

\section{REFERENCES}

[1] V. Mor-Avi, R. M. Lang, and L. P. Badano et al., "Current and evolving echocardiographic techniques for the quantitative evaluation of cardiac mechanics: ASE/EAE consensus statement on methodology and indications endorsed by the Japanese Society of Echocardiography," European Heart Journal, vol. 12, no. 3, pp. 167-205, 2011.

[2] J. U. Voigt, G. Pedrizzetti, and P. Lysyansky et al., "Definitions for a common standard for 2D speckle tracking echocardiography. consensus document of the EACVI/ASE/industry task force to standardize deformation imaging," European Heart Journal, 2014, accepted.

[3] J. D. Thomas and L. P. Badano, "EACVI-ASE-industry initiative to standardize deformation imaging: a brief update from the co-chairs," European Heart Journal, vol. 14, no. 11, pp. 1039-1040, 2013.

[4] M. Alessandrini, H. Liebgott, D. Friboulet and O. Bernard, "Simulation of realistic echocardiographic sequences for ground-truth validation of motion estimation," Proc. of IEEE ICIP, pp. 2329-2332, 2012.

[5] M. Alessandrini, M. De Craene, O. Bernard, et al., "A Pipeline for the Generation of Realistic 3D Synthetic Echocardiographic Sequences: Methodology and Open-access Database," in IEEE $T M I$, accepted.

[6] Hang Gao, Hon Fai Choi, and P. Claus et al., "A fast convolution-based methodology to simulate 2D/3D cardiac ultrasound images," IEEE UFFC, vol. 56, no. 2, pp. 404-409, February 2009.

[7] S. Marchesseau, H. Delingette, and M. Sermesant et al., "Preliminary specificity study of the bestelclmentsorine electromechanical model of the heart using parameter calibration from medical images," JMBBM, vol. 20, pp. 259 - 271, 2013.

[8] A. Papachristidis, M. Geleijnse, and E. Galli et al., "Clinical expert delineation of $3 \mathrm{~d}$ left ventricular echocardiograms for the cetus segmentation challenge," in Proceedings MICCAI Challenge on Endocardial Three-dimensional Ultrasound Segmentation 2014, 2014, pp. 9-16.

[9] M. De Craene, S. Marchesseau, B. Heyde, and H. Gao et al., "3D strain assessment in ultrasound (Straus): A synthetic comparison of five tracking methodologies," IEEE TMI, vol. 32, no. 9, pp. 1632-1646, Sept 2013.

[10] B. Heyde, R. Jasaityte, and D. Barbosa et al., "Elastic image registration versus speckle tracking for $2 \mathrm{D}$ myocardial motion estimation: A direct comparison in vivo," IEEE TMI, vol. 32, no. 2, pp. 449-459, Feb 2013. 